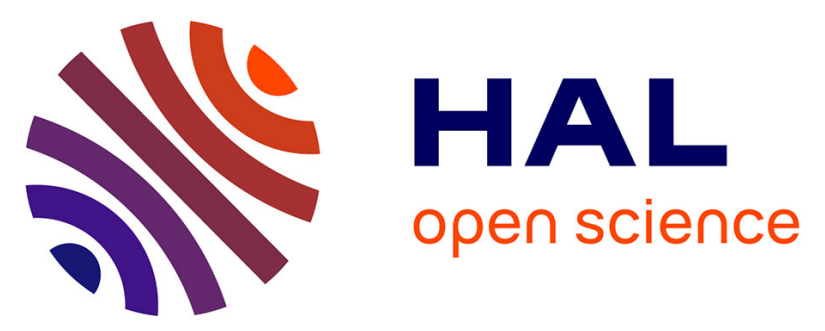

\title{
Accuracy of a CT-Ultrasound Fusion Imaging Guidance System Used for Hepatic Percutaneous Procedures
}

Fabrice Bing, Jonathan Vappou, Elodie Breton, Iulian Enescu, Julien Garnon, Afshin Gangi

\section{- To cite this version:}

Fabrice Bing, Jonathan Vappou, Elodie Breton, Iulian Enescu, Julien Garnon, et al.. Accuracy of a CT-Ultrasound Fusion Imaging Guidance System Used for Hepatic Percutaneous Procedures. JVIR: Journal of Vascular and Interventional Radiology, 2019, 30 (7), pp.1013-1020. 10.1016/j.jvir.2018.11.034 . hal-03088762

\section{HAL Id: hal-03088762 https://hal.science/hal-03088762}

Submitted on 25 Oct 2021

HAL is a multi-disciplinary open access archive for the deposit and dissemination of scientific research documents, whether they are published or not. The documents may come from teaching and research institutions in France or abroad, or from public or private research centers.
L'archive ouverte pluridisciplinaire HAL, est destinée au dépôt et à la diffusion de documents scientifiques de niveau recherche, publiés ou non, émanant des établissements d'enseignement et de recherche français ou étrangers, des laboratoires publics ou privés.

\section{(ㄷ)(1) $\$$}

Distributed under a Creative Commons Attribution - NonCommerciall 4.0 International 


\section{Accuracy of a CT-US fusion imaging guidance system used for hepatic percutaneous procedures}

Fabrice Bing, M.D. ${ }^{1,2,3^{*}}$, Jonathan Vappou, $\mathrm{PhD}^{2}$, Elodie Breton $\mathrm{PhD}^{2}$, Iulian Enescu, M.D. ${ }^{3}$, Julien Garnon, M.D. ${ }^{3}$, Afshin Gangi, $\mathrm{PhD}^{3}$

1 Department of Radiology, Hôpital d'Annecy, 1 avenue de l'Hôpital 74374 Metz-Tessy, France

2 Icube Laboratory, Université de Strasbourg, CNRS, IHU Strasbourg, ICUBE sc IRCAD, 1 place de l'hôpital, 67091, Strasbourg, France

3 Interventional Radiology Department, Hôpitaux Universitaires de Strasbourg, 1 place de l'hôpital, 67091, Strasbourg, France

* corresponding author:

Fabrice Bing

Mail: fabricebing@yahoo.fr

tel (+33) 450637007

fax (+33) 450636083

Conflict of interest: none

Acknowledgment: The FUS evaluated was gift from Toshiba. We thank Dr Sauleau ErikAndré (Pôle de Santé Publique, Groupe Méthodologique en Recherche Clinique, Hôpital Civil, Strasbourg) who performed the statistical analysis. 


\section{Accuracy of a CT-US fusion imaging guidance system used for hepatic percutaneous procedures}

Abstract:

Objectives: To evaluate the accuracy of a Fusion imaging guidance system using US and CT as a real-time imaging modality for the positioning of a 22-gauge needle in the liver.

Materials and Methods: The spatial coordinates of 23 spinal needles placed at the border of hepatic tumors before radiofrequency thermal ablation were determined in 23 patients. Needles were inserted up to the border of the tumor using CT-US fusion imaging. A control CT-scan was carried out to compare real (x, y, z) and virtual (x', y', z') coordinates of the tip of the needle ( $\mathrm{D}$ for distal) and of a point on the needle located $3 \mathrm{~cm}$ proximally to the tip (P for proximal).

Results: The mean Euclidian distance was $8.5 \pm 4.7 \mathrm{~mm}$ and $10.5 \pm 5.3 \mathrm{~mm}$ for D and $\mathrm{P}$ respectively. The absolute value of mean differences of the three coordinates $\left(|x '-x|,\left|y^{\prime}-y\right|, \mid z^{\prime}-\right.$ $\mathrm{z} \mid$, mean $\pm \mathrm{SD}$ ) were $4.06 \pm 0.9,4.21 \pm 0.84,4.89 \pm 0.89 \mathrm{~mm}$ for point $\mathrm{D}$ and $3.96 \pm 0.60$ $4.41 \pm 0.86,7.66 \pm 1.27 \mathrm{~mm}$ for point $\mathrm{P} . \mathrm{X}=\left|\mathrm{x}^{\prime}-\mathrm{x}\right|$ and $\mathrm{Y}=\left|\mathrm{y}^{\prime}-\mathrm{y}\right|$ coordinates were less than $7 \mathrm{~mm}$ with a probability close to $1 . Z=\left|z^{\prime}-z\right|$ coordinate was not considered larger or smaller than 7 $\mathrm{mm}$ (Probability $>7 \mathrm{~mm}$ close to $50 \%)$.

Conclusions: Positioning errors using the US-CT Fusion imaging used in this study are not negligible for the insertion of a 22-gauge needle in the liver. Physicians must be aware of such possible errors in order to adapt the treatment when used for thermal ablation.

Keywords:

Fusion imaging; Hepatic tumor; Ultrasonography, Interventional; Ablation Techniques. 
Introduction

Percutaneous interventional procedures need accurate device positioning, using imaging guidance such as ultrasound (US), Computed Tomography (CT), fluoroscopy or Magnetic Resonance Imaging (MRI). Fusion imaging guidance systems (FIGS) may be an interesting solution when the interventional radiologist needs a combination of information provided by two or more imaging modalities. This can be the case for the positioning of the needle (biopsy) or for the evaluation of the ablative margins, for instance in radiofrequency ablation (RFA) for malignant hepatic tumors [1-4]. Virtual navigation systems in preoperative 3D MRI or CT datasets bring the possibility of improving real-time tumor targeting. These systems can be used alone with optical or electromagnetic tracking platforms or associated with US guidance [5]. With this second solution, the operator can navigate within the pre-operative CT or MRI 3D volume superimposed with the real-time US exploration. Hence, FIGS bring the possibility of navigating in an enhanced 3D volume, such as contrast enhanced CT or MRI (arterial or portal vein phase is chosen depending on which one allows the best visibility of the hepatic lesion). The operator can at any time during the procedure navigate through the $3 \mathrm{D}$ contrast enhanced volume chosen, even if contrast enhancement is time-limited. However, the operator has to be sure that when considering the virtual volume, it corresponds to reality. This is even more important in case of tumors difficult to visualize with ultrasound [6-9].

The evaluation of accuracy of FIGS using US as real-time imaging modality for positioning a needle in vitro [10] or in a mobile organ [11] has been scarcely reported. The objective of the present study was to investigate the accuracy of this system by measuring the distance between virtual (where the needle is assumed to be considering the fused navigation 
$\mathrm{CT}$ ) and real (where the needle is on the control CT-scan) locations of a 22-gauge needle put in contact with a hepatic tumor.

Materials and Methods

This prospective study was approved by the local Institutional Review Board (IRB). Patient anonymity was preserved and the principles of the Declaration of Helsinki were followed. Informed consent was obtained from all patients. Twenty-five patients were included consecutively. Inclusion and exclusion criteria are listed in Table 1. Two patients were excluded. One patient was excluded because the fusion was performed with MRI, which resolution is too low compared to the one of CT-scan. Another patient was excluded for technical reasons (fusion system not available). The two operators who performed the ablations had 6 and 7 years of experience in hepatic tumor radiofrequency ablation under US guidance respectively, and 2 years of experience with the FIGS evaluated in this study. The ablations were performed in the Department of Interventional Radiology (University Hospital, 25 years of experience in oncologic interventional radiology).

Twenty-three needles in 23 patients (mean age \pm SD, $67.6 \pm 8.1$ years) treated by RFA for hepatocellular carcinoma (HCC) were evaluated. The evaluation of the accuracy of the imaging system was performed during the first step of the treatment, which consisted in the insertion of a 22-gauge spinal needle (Chiba biopsy needle; Cook, Bloomington, Ind) in the liver using the CT-US fusion imaging system (Toshiba, TUS-A500, SmartFusion application). This step aimed at positioning the tip of the needle at the external border of the tumor, without penetrating it, based on the real-time echography guidance alone. The border of the tumor is defined as the first contact of the spinal needle with the limit of the tumor seen on echography (Fig. 1). The aim was to evaluate the accuracy of the system using a very fine needle that would not injure the organs, as opposed to the larger final needle used for RFA 
(Cool-tip ${ }^{\mathrm{TM}}$ RFA system, Medtronic, Minneapolis, USA). The pathway of this spinal needle was later used for the insertion of the radiofrequency needle, but this step does not concern the evaluation.

The system evaluated in our study uses electromagnetic tracking and is composed of a tracking workstation and a magnetic field generator. Multimodality image fusion is possible with that system but in this study, only a CT-scan performed on the operative table, was employed for fusion. In brief, a magnetic position sensor was attached to the ultrasound transducer shaft. A CT-scan (16-detector row scanner, Somatom; Siemens, Healthcare GmbH, Forchheim, Germany) was performed just before the procedure and 3D Digital imaging and Communications in Medicine (DICOM) datasets were used for fusion. Scan parameters were as follows: detector collimation, $32 \times 1.2 \mathrm{~mm}$; reconstruction thickness, $3 \mathrm{~mm}$; scan pitch, $0.8^{\circ}$; and gantry rotation speed, 0.5 seconds. General anesthesia was performed before the CT-scan acquisition and the patient remained in the same position on the CT table during the whole procedure.

The coregistration procedure was performed according to the manufacturer guidelines, detailed in the following. The fusion points chosen presented two characteristics: first, it had to be well identified on the CT-scan and with US; second, it had to present the same movement as the tumor, which means that it has to be ideally situated next to the target. Some examples are the portal vein (at the right and left bifurcation), the origin of one hepatic vein, the origin of the celiac trunk or the gallbladder's infundibulum. A liver cyst can also be used if easily seen on both modalities. The quality index of the fusion indicated by the system depends on the distance between the probe (magnetic position sensor put on the left side of the patient) and the magnetic field generator. After defining an initial anatomic landmark, the operator navigates through the fused volumes in order to evaluate the quality of the fusion. If the fusion is judged correct by the operator and one technician, the coregistration procedure is 
stopped. If not, new reference points are added until the operator considers the coregistration satisfying. The choice of the anatomic points depends on the echogenicity, which is patient dependant. For each patient, the operator made the effort to be as precise as possible, in order to minimize operator dependant errors in registration.

Each point is defined by its Cartesian coordinates $(\mathrm{x}, \mathrm{y}, \mathrm{z})$ in the three-dimensional Euclidean space (Fig. 1). XY-plane is the transversal plane, X corresponding to the left-right axis (length) and $\mathrm{Y}$ to the anteroposterior axis (depth). $\mathrm{Z}$ is the craniocaudal axis (height). To calculate the real coordinates of the needle, a CT-scan was repeated once the spinal needle was in its final position and at the end-expiratory phase, before the insertion of the radiofrequency needle. Differences between real $(x, y, z)$ and virtual (x', y,' z') coordinates of the tip of the needle ( $\mathrm{D}$ for distal point) and of a needle point located $3 \mathrm{~cm}$ proximally to the tip (P for proximal) were determined (Fig. 1).

To obtain the virtual coordinates, the CT plane seen on the US fusion had to be exactly reproduced using the initial 3D CT volume (Figs. 2 and 3). The virtual location of the tip of the needle was then carried over to the CT volume and the coordinates ( $\left.\mathrm{x}^{\prime}, \mathrm{y}^{\prime}, \mathrm{z}^{\prime}\right)$ of the points $\mathrm{P}$ and $\mathrm{D}$ were calculated, using the same geometrical construction elaborated on the virtual scanner and US image showed on figure 2 . The real coordinates were easily calculated on the control scan (Fig. 4).

For each case, differences between real and virtual coordinates of $\mathrm{D}$ and $\mathrm{P}$ on the three axis, defined as $X=\left|x^{\prime}-x\right|, Y=\left|y^{\prime}-y\right|, Z=\left|z^{\prime}-z\right|$ respectively, was calculated. For each patient, the global mean error $\left(\frac{\left(X_{P}+Y_{P}+Z_{P}+X_{D}+Y_{D}+Z_{D}\right)}{6}\right)$ was calculated. Mathematically, the Euclidian distance is the Cartesian version of Pythagoras's theorem [12]. The distance (RV) between R for real $(X, Y, Z)$ and $V$ for virtual $\left(X^{\prime}, Y^{\prime}, Z^{\prime}\right)$ in three-dimensional space is given by the formula: $\sqrt{\left(x-x^{\prime}\right)^{2}+\left(y-y^{\prime}\right)^{2}+\left(z-z^{\prime}\right)^{2}}=\sqrt{X^{2}+Y^{2}+Z^{2}}$. The Euclidean distance is 
the best metric for representing the distance between two points in a three-dimensional space since it takes into account all of the three components of points position (X,Y,Z) equally [12].

The coordinates of the needle were calculated in the volume space of the operative room. Pre-procedural and control CT-scan were performed with the anesthesia ventilator stopped (end-respiratory pause). The operator inserted the needle and recorded its final position when the patient was at the end-expiratory phase. However, as the respiratory pause may not be exactly the same, the coordinates of the hepatic tumor and by extrapolation the needle between the pre-procedural and the control CT-scan may change. In consequence, the coordinates of the needle were calculated in the good referential taking into account movements of the liver. For this, the differences of coordinates of a liver point (A) easily recognized on both scans performed before $\left(\mathrm{x}_{\mathrm{A}}, \mathrm{y}_{\mathrm{A}}, \mathrm{z}_{\mathrm{A}}\right)$ and after the needle insertion $\left(\mathrm{x}_{\mathrm{A}}{ }_{\mathrm{A}}\right.$, $\mathrm{y}^{\prime}{ }_{\mathrm{A}}, \mathrm{z}^{\prime} \mathrm{A}$ ), were calculated. The liver point (A) may correspond to an anatomic structure next to the tumor. Movement of the patients was not considered here as all the patients were under general anesthesia and remained in the same position on the table. The difference between the initial and the control coordinates of (A) was used to correct those of the needle. Hence, the real coordinates of the needle calculated on the final CT-scan were rectified in the new reference, that was the liver.

For each case, the duration of the procedure (delay between the installation of the FIGS and the CT-scan control), the distance between the skin entry point and the tumor, the diameter of the tumor, the quality of the fusion (scale between 1 (very bad) and 10 (very good)) and the satisfaction of the operator (scale between 1 (not satisfied at all) to 5 (very satisfied)) were determined. The quality index (1 to 10) indicated by the manufacturer on the screen of the system (Fig. 4), depends on the intensity of the electromagnetic signal recorded by the system. 


\section{Statistical analysis}

The aim of the study was to estimate a distance within its interval of credibility. To determine the sample size, a mean error $\mu=5 \mathrm{~mm}$ of accuracy for each axis, was considered with a standard deviation $\sigma=2.5 \mathrm{~mm}$. A Bayesian analysis of the data (software R, OpenBUGS) was performed to know the probability of the error (difference between real and virtual coordinates). The estimation determined a minimal sample size of 20 patients, which was increased to 25 in this study. No interim analysis was scheduled. After the inclusion of the last patient, the Bayesian analysis was performed for $\mu=5$ and $\mu=7 \mathrm{~mm}$. The differences were compared to $7 \mathrm{~mm}$ by using gamma distributions and Bayesian methods. The relationship between the quality of the fusion, the depth of the lesion, and the global mean error was studied using Spearman's correlation coefficient.

Results

Mean tumor's diameter was $22.6 \pm 8.6 \mathrm{~mm}$ for a mean depth (skin-tumor distance) of $90.1 \pm 30.8 \mathrm{~mm}$ (Table 2). Mean differences of (A) coordinates were $1.8 \pm 1.8 \mathrm{~mm}, 2.1 \pm 2.0 \mathrm{~mm}$ and 3.8 $\pm 4.3 \mathrm{~mm}$ for $\left|\mathrm{x}_{\mathrm{A}}{ }^{\prime}-\mathrm{x}_{\mathrm{A}}\right|,\left|\mathrm{y}_{\mathrm{A}}{ }^{\prime}-\mathrm{y}_{\mathrm{A}}\right|,\left|\mathrm{z}_{\mathrm{A}}{ }^{\prime}-\mathrm{z}_{\mathrm{A}}\right|$ respectively. The absolute value of mean differences of the three coordinates $\left(\left|x^{\prime}-x\right|,\left|y^{\prime}-y\right|,\left|z^{\prime}-z\right|\right.$, mean $\left.\pm S D\right)$ were $4.06 \pm 0.9,4.21 \pm 0.84$, $4.89 \pm 0.89 \mathrm{~mm}$ for point $\mathrm{D}$ and $3.96 \pm 0.60,4.41 \pm 0.86,7.66 \pm 1.27 \mathrm{~mm}$ for point $\mathrm{P}$ (Fig. 5). The $\mathrm{X}$ and $\mathrm{Y}$ coordinates were less than $7 \mathrm{~mm}$ with a probability close to $1 . \mathrm{Z}$ coordinates were not considered to be larger or smaller than $7 \mathrm{~mm}$ (Probability $>7 \mathrm{~mm}$ close to $50 \%$ ). The mean Euclidean distance $\left(\sum \sqrt{ }\left(\left(x i^{\prime}-x i\right)^{2}+(y i '-y i)^{2}+(z i '-z i)^{2}\right) / 23\right)$ between virtual and real coordinates was $8.5 \pm 4.7 \mathrm{~mm}$ and $10.5 \pm 5.3 \mathrm{~mm}$ for point $\mathrm{D}$ and P respectively (Fig. 6).

No correlation was observed between the depth of the tumor and the global mean error for point $\mathrm{P}$ and $\mathrm{D}(\rho=-0.07)$. The mean duration of the procedure was $14.7+/-10 \mathrm{~min}$. The use 
of the fusion system increased the duration of the procedure for cases 6 and 8 . In these cases, fusion procedure took more time as orientation in space (cranio-caudal inversion) was not correct. This required restarting the initial step of fusion which delayed the procedure.

Rigid coregistration was performed using one to five (mean $2.2 \pm 1.2$ ) internal anatomic landmarks (portal vein, celiac artery, hepatic veins) to spatially match the two imaging datasets to each other. No correlation between the index of quality and the global mean error $(\rho=0.097)$ was observed.

Globally, the operators were satisfied with the fusion system (mean of 3.75/5) and used the system for the positioning of the final needle (radiofrequency needle). No learning curve effect was reported, as operators did not have any feedback on the accuracy of the fusion system during the study.

\section{Discussion}

The accuracy of a fusion imaging guidance system for the insertion of a 22-gauge needle in the liver was evaluated. The FIGS evaluated allows navigation in both ultrasound and preoperatively acquired CT volumes with a mean difference in Euclidian coordinates of $8.5 \pm 4.7$ $\mathrm{mm}$ and $10.5 \pm 5.3 \mathrm{~mm}$ for the tip of the needle and a needle point located $3 \mathrm{~cm}$ proximally, respectively. The error measured corresponds firstly to the registration error, secondly to the error due to the localized deformation of the organ and thirdly to liver motion during breathing (even if precautions have been taken to register the data in expiration). Moreover, an operator error may be added, as the needle is not seen on the virtual CT-scan: the operator extrapolates the localization of the needle seen with US on the CT-scan picture. The aim was not to report a clinical error, as the tip of the needle was not positioned at the center of the tumor. 
Different fusion systems using optical, electromagnetic and cone beam CT-scan have been evaluated and good accuracy of these systems has been reported [13]. The use of FIGS is very interesting when the operator needs information from previous imaging modalities such as positron emission tomography (PET), MRI or contrast-enhanced CT. The interest of the multimodality image fusion is greater when the tumor is only seen on arterial or venous phase CT or MRI: in this situation, the operator can see a tumor not visible with one modality alone (US or non-enhanced CT) and can "fix" the enhancement of the tumor for the time of the procedure. Moreover, even if the tumor is seen with US, only one part may be the target for percutaneous biopsy or destruction. Fusion with PET scan showing the metabolic activity of the tumor may be helpful in these cases. In our study, the operator was able to see the lesion with US but the lesion was hardly identified or not visible on the unenhanced CT-scan. In fact, the partial visibility of the lesion was reproduced which corresponds to the real application and interest of the fusion system.

In previous studies, the overall error measured corresponds to the distance between the needle tip and the center of the tumor $[5,10,11,14-16]$. In fact, the problem with the distance of the needle tip to the tumor is that the target may be large (as in our study with a mean tumor diameter of $22.6 \mathrm{~mm}$ ): the system may be considered as being accurate for larger tumors but not for small ones. Moreover, the tip of the needle can be in the tumor on the CTscan control and considered as being well placed, although the operator may have thought to be in a different location when performing the procedure.

Using the distance of the needle tip to the target center, the accuracy of electromagnetic systems in biopsy and ablation procedures has been reported as being clinically acceptable $[17,18]$. Using an electromagnetic system, a spatial accuracy of $3.1 \pm 2.1$ $\mathrm{mm}$ has been reported for image-guided interventions [18]. Authors of this article reported a significant decrease of the radiation dose when using the electromagnetic system. With a CT- 
US imaging guiding system using electromagnetic tracking, the mean registration error reported was $3.0 \pm 0.1 \mathrm{~mm}$ using calf livers [10]. In a study using CT imaging alone with electromagnetic needle tracking, the image registration error was $1.4 \mathrm{~mm}$ in the phantom but $19 \mathrm{~mm}$ in the animal [17]. The accuracy of CT-US image system was evaluated in pigs: from $6 \mathrm{~mm}$ for muscle and kidney, the accuracy dropped to $14 \mathrm{~mm}$ in the liver [19]. This discrepancy emphasizes the problem of respiratory motion induced error. For an optimal navigation in the US 3D volume, the operator needs to exert a pressure on the abdomen with the probe that may stretch the liver in all three dimensions. This deformation is not taken into account in the CT volume (rigid registration) and the operator has to adapt this pressure to understand the location of the needle in the virtual 3D CT volume. The liver motion during breathing results in a modification of the real position of the target, which is not shown in the virtual CT volume [20]. Initial, final CT-scan and procedure were performed in an ideal situation as the patient was exactly in the same position on the table. With the system evaluated in our series, there was no need of cutaneous fiducial skin markers for the registration, but the operator had to choose anatomic landmarks visible on CT and with US. This step is essential, and the operator has to multiply the search of fusion points. Ideally, the anatomic landmarks have to be fused exactly at the same level and during the same breathing cycle of the initial scan. For right intercostal access, the fusion quality index was systematically low, situated between 4 and 5, whereas the quality index of the fusion point was higher (between 8 and 10). This is explained by the remoteness from the electromagnetic field generator which has to remain fixed for all the procedures. When the index was low $(<5)$, the navigation in the CT volume was less fluid (the system had tendency to display the image with a delay of few ms when moving the transducer). However, no correlation between the quality of the fusion indicated by the system and the precision was found. 
The Euclidian distance (about $9 \mathrm{~mm}$ in our work) between real and virtual points is mathematically the most appropriate way to report 3D spatial distances, but this measurement has never been used in the literature for clinical evaluations of fusion systems. This error is substantial and may impact the quality of the ablation, particularly concerning the margins. However, the operators reported to be confident with the system and the mean duration (delay between the installation of the FIGS and the CT-scan control) was 14 min, which is correct. The error measured should be reevaluated with the insertion of the final needle that may further modify the anatomy and when the prior MRI or CT 3D volume is performed away from the procedure. This situation is closer to real everyday examinations. The interventional radiologist must be aware of potential discrepancies between the reality and the virtual information given by the fusion system, that may have an impact on ablative margin or even the safety of the treatment [21].

This study presents several limitations. Firstly, the reconstruction of the virtual CT plane added a supplementary error. It is difficult to quantify the reproducibility of the virtual plane. Secondly, the needle was not visible on the virtual CT-scan and an extrapolation of the tip and angulation of the needle was made from the US image to the virtual CT-scan. However, this extrapolation is mentally used by the operator when performing the puncture and the same process was used for the measurement. Thirdly, manual registration may be changed for automatic image fusion techniques as positioning and sweeping auto-registration. These techniques may improve the quality of the fusion [2]. Fourthly, the error was determined in ideal conditions as the CT-scan recorded was performed just before the procedure in the exact position of the puncture. Even if the ventilator was not halted during the insertion of the needle, the patient was under general anesthesia and the insertion of the needle was systematically performed at the end-expiratory phase. It would be interesting to evaluate the accuracy of the system when the loaded CT-scan or MRI is performed several 
days before the puncture. Finally, the system has been upgraded with "needle-tracking" which offers the opportunity to see virtually the needle on the CT-scan. This option has not been used in this study. This option may help for the visualization of the needle even if it does not take into account the deformation of the needle. It may be more challenging to practice fusion for other locations where anatomic landmarks may be difficult to find (lung, peripheral bone and articulations for example).

In conclusion, the results show that errors between what the operator sees on the virtual CT-scan and where the needle actually is on the control CT-scan can be substantial. In the situation of hepatic tumors that are difficult to visualize, FIGS may be very helpful, but the interventional radiologist has to be aware of a potential discrepancy between real and virtual coordinates of the needle. It is recommended to practice intermediate CT-scans to confirm proper positioning of the probe. It may be necessary to insert two probes to compensate the potential inaccuracies in order to obtain sufficient margins.

\section{References}

[1] Abdel-Rehim M, Ronot M, Sibert A, et al. Assessment of liver ablation using cone beam computed tomography. World J Gastroenterol. 2015;21:517-524.

[2] Cha DI, Lee MW, Kim AY, et al. Automatic image fusion of real-time ultrasound with computed tomography images: a prospective comparison between two auto-registration methods. Acta Radiol. 2017;58:1349-1357.

[3] $\mathrm{Li} \mathrm{K}, \mathrm{Su} \mathrm{Z}, \mathrm{Xu} \mathrm{E}$, et al. Evaluation of the ablation margin of hepatocellular carcinoma using CEUS-CT/MR image fusion in a phantom model and in patients. BMC Cancer. 2017;17:61. 
[4] Minami Y, Minami T, Hagiwara S, et al. Ultrasound-ultrasound image overlay fusion improves real-time control of radiofrequency ablation margin in the treatment of hepatocellular carcinoma. Eur Radiol. 2018;28:1986-1993.

[5] Krucker J, Xu S, Venkatesan A, et al. Clinical utility of real-time fusion guidance for biopsy and ablation. J Vasc Interv Radiol. 2011;22:515-524.

[6] Hirooka M, Iuchi H, Kumagi T, et al. Virtual sonographic radiofrequency ablation of hepatocellular carcinoma visualized on CT but not on conventional sonography. AJR Am J Roentgenol. 2006;186:S255-60.

[7] Makino Y, Imai Y, Ohama H, et al. Ultrasonography fusion imaging system increases the chance of radiofrequency ablation for hepatocellular carcinoma with poor conspicuity on conventional ultrasonography. Oncology. 2013;84 Suppl 1:44-50.

[8] Lee MW, Rhim H, Cha DI, et al. Percutaneous radiofrequency ablation of hepatocellular carcinoma: fusion imaging guidance for management of lesions with poor conspicuity at conventional sonography. AJR Am J Roentgenol. 2012;198:1438-1444.

[9] Nakai M, Sato M, Sahara S, et al. Radiofrequency ablation assisted by real-time virtual sonography and CT for hepatocellular carcinoma undetectable by conventional sonography. Cardiovasc Intervent Radiol. 2009;32:62-69.

[10] Crocetti L, Lencioni R, Debeni S, et al. Targeting liver lesions for radiofrequency ablation: an experimental feasibility study using a CT-US fusion imaging system. Invest Radiol. 2008;43:33-39.

[11] Hakime A, Deschamps F, De Carvalho EG, et al. Clinical evaluation of spatial accuracy of a fusion imaging technique combining previously acquired computed tomography and 
real-time ultrasound for imaging of liver metastases. Cardiovasc Intervent Radiol. 2011;34:338-344.

[12] Hughes-Hallett D, McCallum W, Gleason AM. Calculus : Single and Multivariable. 6th ed. John Wiley; 2012.

[13] Abi-Jaoudeh N, Kruecker J, Kadoury S, et al. Multimodality image fusion-guided procedures: technique, accuracy, and applications. Cardiovasc Intervent Radiol. 2012;35:986-998.

[14] Appelbaum L, Solbiati L, Sosna J, et al. Evaluation of an electromagnetic image-fusion navigation system for biopsy of small lesions: assessment of accuracy in an in vivo swine model. Acad Radiol. 2013;20:209-217.

[15] Appelbaum L, Sosna J, Nissenbaum Y, et al. Electromagnetic navigation system for CTguided biopsy of small lesions. AJR Am J Roentgenol. 2011;196:1194-1200.

[16] Penzkofer T, Bruners P, Isfort P, et al. Free-hand CT-based electromagnetically guided interventions: accuracy, efficiency and dose usage. Minim Invasive Ther Allied Technol. 2011;20:226-233.

[17] Santos RS, Gupta A, Ebright MI, et al. Electromagnetic navigation to aid radiofrequency ablation and biopsy of lung tumors. Ann Thorac Surg. 2010;89:265-268.

[18] Wood BJ, Zhang H, Durrani A, et al. Navigation with electromagnetic tracking for interventional radiology procedures: a feasibility study. J Vasc Interv Radiol. $2005 ; 16: 493-505$. 
[19] Banovac F, Wilson E, Zhang H, et al. Needle biopsy of anatomically unfavorable liver lesions with an electromagnetic navigation assist device in a computed tomography environment. J Vasc Interv Radiol. 2006;17:1671-1675.

[20] Spinczyk D. Towards the clinical integration of an image-guided navigation system for percutaneous liver tumor ablation using freehand 2D ultrasound images. Comput Aided Surg. 2015;20:61-72.

[21] Makino Y, Imai Y, Igura T, et al. Usefulness of the multimodality fusion imaging for the diagnosis and treatment of hepatocellular carcinoma. Dig Dis. 2012;30:580-587.

Table and figures legends:

Table 1. Inclusion and exclusion criteria.

Table 2. Details of the results for each patient. Patient's numbers correspond to a chronological order. *delay between the installation of the FIGS and the CT-scan control.

Figure 1. Drawing showing the real points $D_{R}$ and $P_{R}$ on the spinal needle (full line $R$ ) and the virtual points $D_{V}$ and $P_{V}$ on the virtual needle (dotted line $V$ ). $D_{R}$ and $D_{V}$ correspond to the tip of the needle. $\mathrm{P}_{\mathrm{R}}$ and $\mathrm{P}_{\mathrm{V}}$ correspond to a point located $3 \mathrm{~cm}$ proximally from $\mathrm{D}$ on the spinal needle. The target (point (b) for border) corresponds to the entry point of the needle in the tumor. The center (c) of the tumor is situated on the needle's path. For each point, the 
coordinates in the three-dimensional space of the real and virtual points are compared and used to determine the accuracy of the system.

Figure 2. Virtual CT image (left side) and corresponding real-time US image (right side) used for the insertion of the 22-gauge needle in the liver. The distance between the tip of the needle (white point in the circle in the US image) and the liver capsula and angulation between the needle and the tangent of the capsula are calculated on the US image and extrapolated in the CT image.

Figure 3. Reconstruction of the virtual CT plane with the initial CT volume to obtain virtual coordinates of the tip of the needle ( $\left.\mathrm{D}^{\prime}\left(\mathrm{x}^{\prime}, \mathrm{y}^{\prime}, \mathrm{z}^{\prime}\right)\right)$ and a point on the needle $3 \mathrm{~cm}$ proximally from the tip ( $\left.\mathrm{P}^{\prime}\left(\mathrm{x}^{\prime}, \mathrm{y}^{\prime}, \mathrm{z}^{\prime}\right)\right)$. All organs seen on the picture have to present the same aspect as on the virtual CT plane (Figure 2, left side for comparison). Once the good plane obtained the same angulation and distance of the needle in the liver as seen on the real US image are reported in the plane and on the virtual coordinates calculated.

Figure 4. Control CT-scan showing the spinal needle. Proximal (P) and distal (D) points correspond to real coordinates of the spinal needle.

Figure 5. Graphic representation of the mean error for $\mathrm{X}, \mathrm{Y}$ and $\mathrm{Z}$ for the distal (D) and the proximal $(\mathrm{P})$ point. $\mathrm{X}$-axis: mean error $(\mathrm{mm})$; Y-axis: number of patients. 
Figure 6. Graphic representation of the mean Euclidean distance between virtual and real coordinates. X-axis: mean Euclidian error (mm); Y-axis: number of patients. 


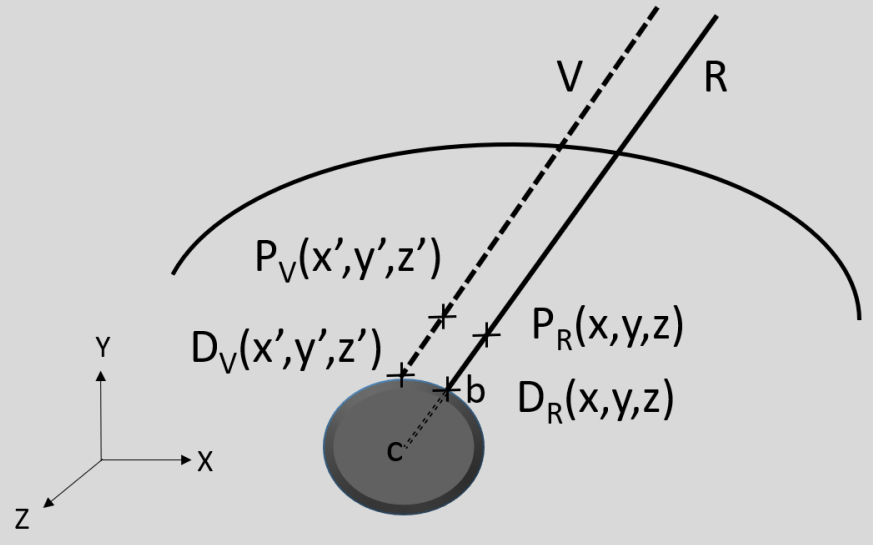



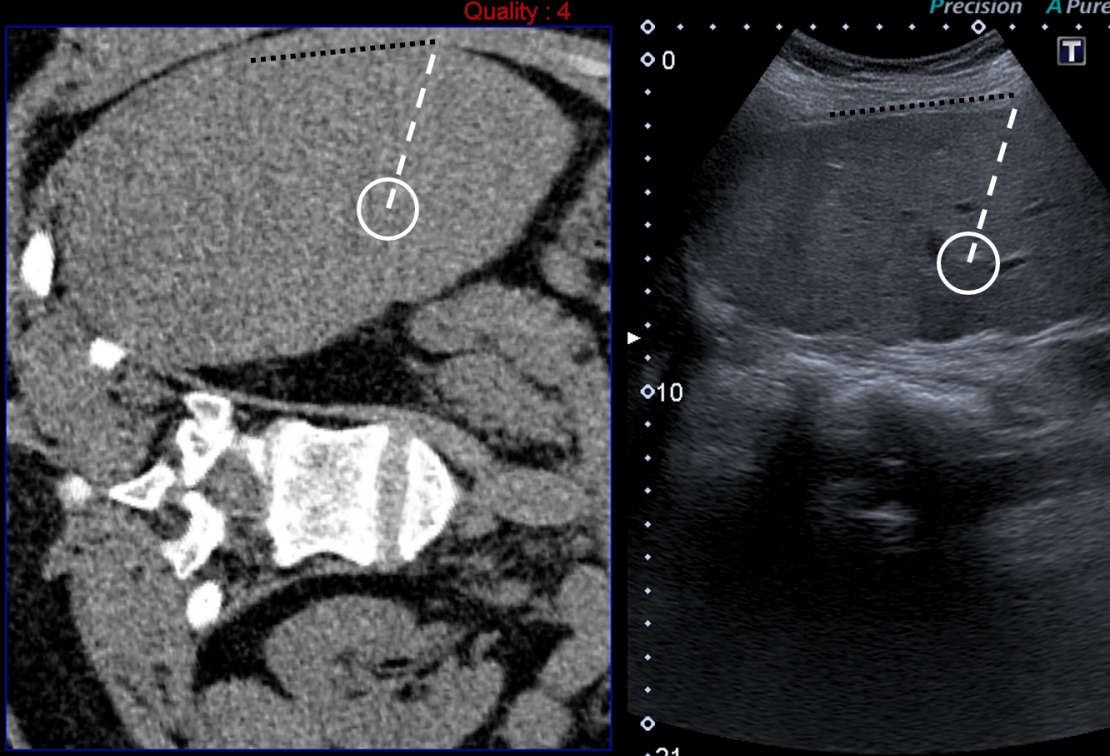

$6 \mathrm{Cl}$ 010

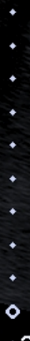
diffT 5.0 13 fps Qscan G:82 DR:65

$A: 2$
$P: 3$ 


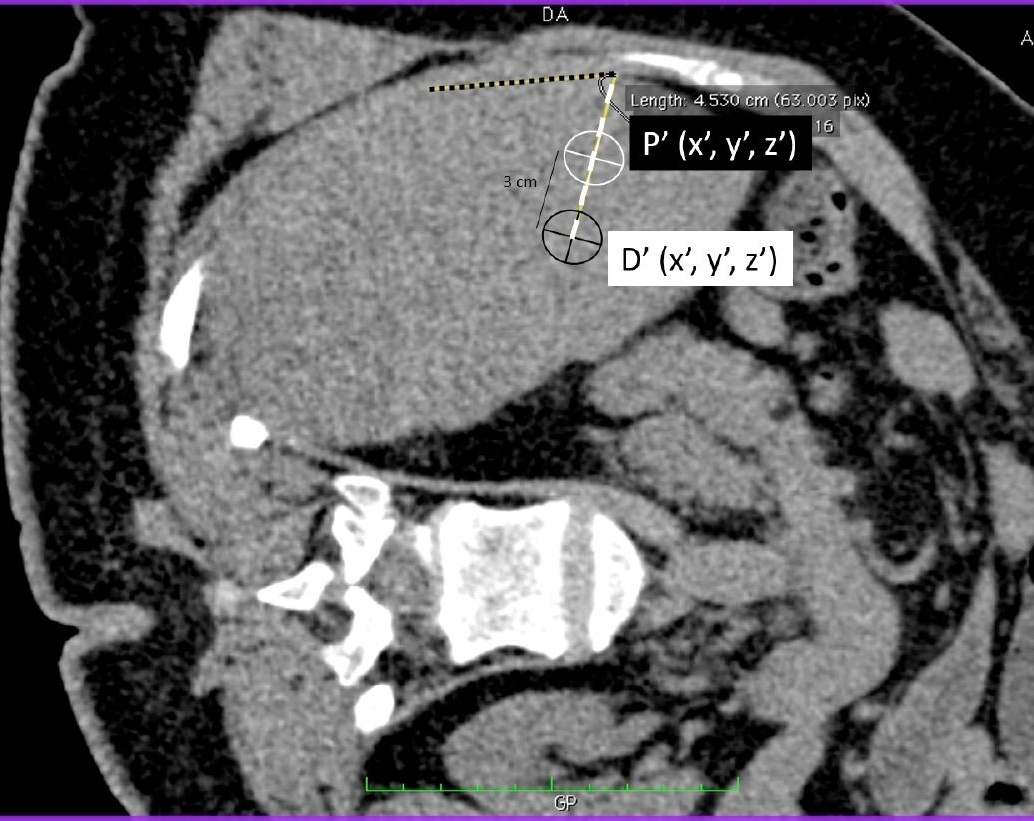




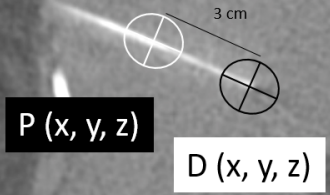

Zoom: $248 \%$ Angle : 179

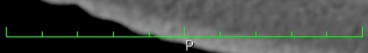



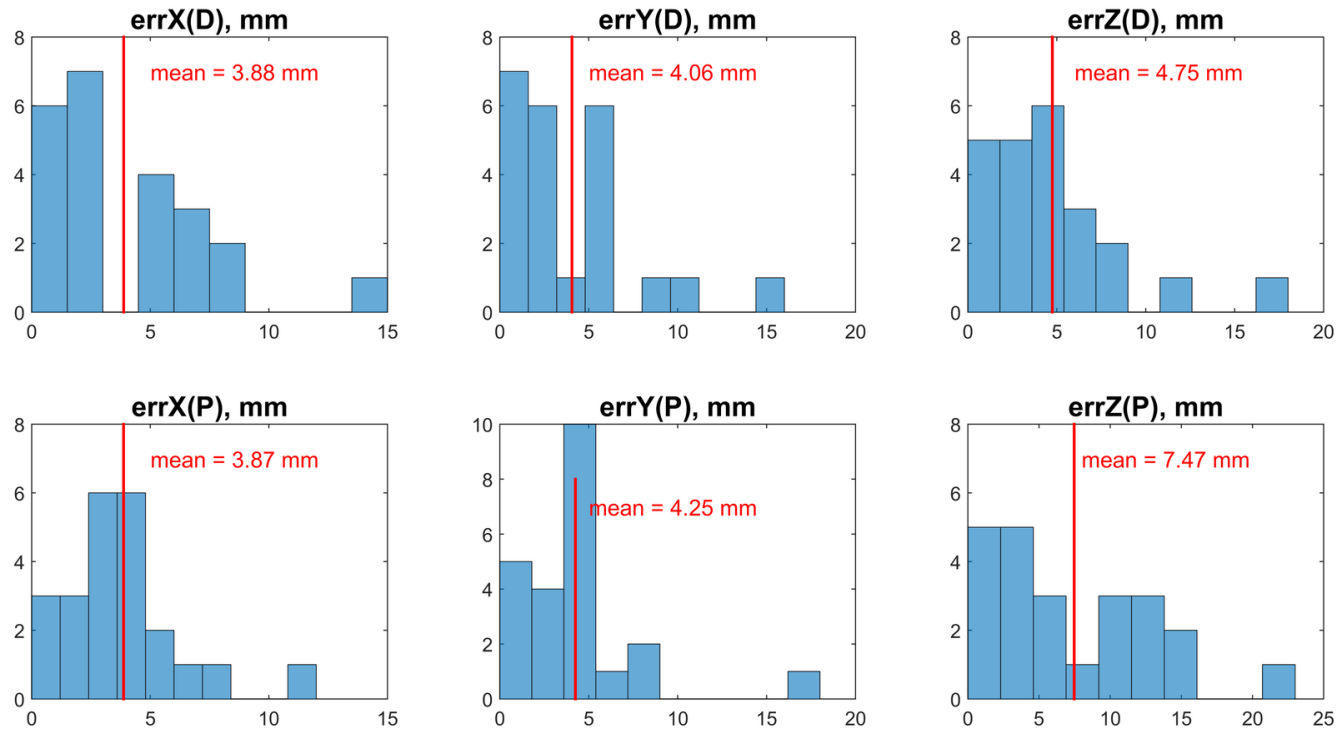


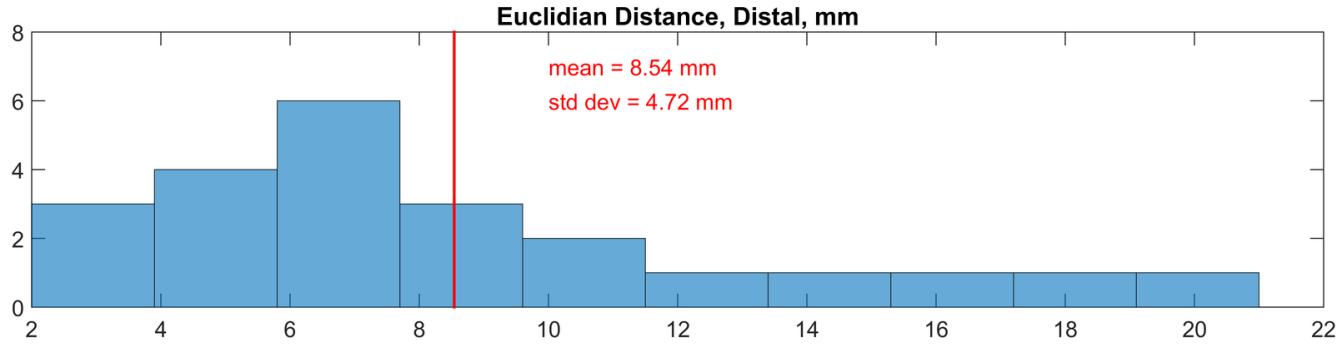

Euclidian Distance, Prox, mm

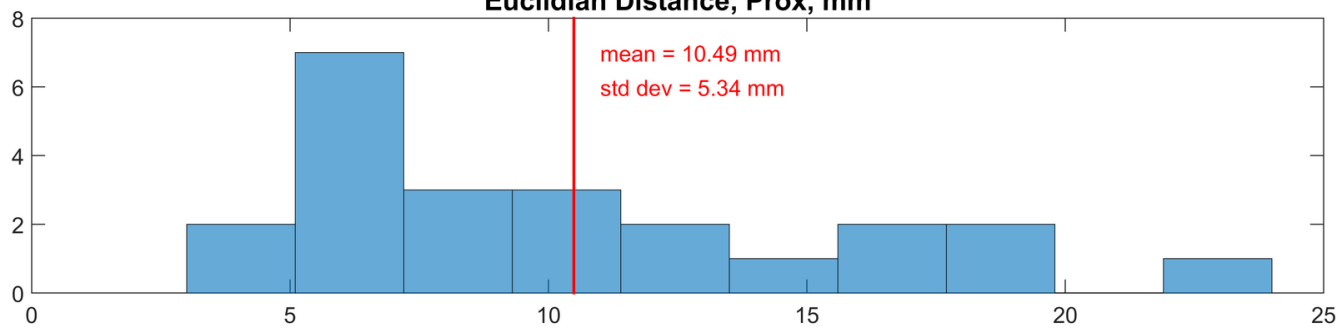


Inclusion criteria

Age>18-year

Patient with primary or secondary hepatic

tumor for which a radiofrequency ablation is possible

Patient who received clear explanation of the radiofrequency and the study during a consultation with an interventional radiologist

Patient who signed the informed consent for the ablation and the inclusion in the study CT-scan performed before the fusion and after the insertion of the 22-G needle

\section{Exclusion criteria}

Pregnancy (contra-indication to X-ray exposure)

Heart stimulator or defibrilator (contraindication to the system's electromagnetic field exposure)

Patient under tutorship

Contraindications to the radiofrequency treatment 


\begin{tabular}{|l|c|c|c|c|c|c|}
\hline Patient & $\begin{array}{c}\text { Diameter } \\
\text { of the } \\
\text { lesion } \\
(\mathrm{mm})\end{array}$ & $\begin{array}{c}\text { Depth of } \\
\text { the lesion } \\
(\mathrm{mm})\end{array}$ & $\begin{array}{l}\text { Duration of } \\
\text { the } \\
\text { procedure* } \\
\text { (min) }\end{array}$ & $\begin{array}{c}\text { Number of } \\
\text { points used } \\
\text { for the } \\
\text { registration }\end{array}$ & $\begin{array}{c}\text { Quality of } \\
\text { the fusion } \\
\text { (according to } \\
\text { the system) }\end{array}$ & $\begin{array}{c}\text { Global mean error } \\
\text { for P and D (mm) }\end{array}$ \\
\hline 1 & 22 & 89 & 20 & 1 & 9 & 3,0 \\
\hline 2 & 16 & 82 & 10 & 1 & 10 & 4,8 \\
\hline 3 & 41 & 89 & 11 & 2 & 5 & 3,3 \\
\hline 4 & 35 & 70 & 10 & 2 & 4 & 2,4 \\
\hline 5 & 40 & 75 & 8 & 2 & 7 & 10,1 \\
\hline 6 & 18 & 120 & 40 & 1 & 8 & 4,2 \\
\hline 7 & 22 & 80 & 15 & 3 & 4 & 4,0 \\
\hline 8 & 16 & 150 & 45 & 3 & 4 & 2,3 \\
\hline 9 & 12 & 80 & 7 & 1 & 7 & 4,6 \\
\hline 10 & 13 & 128 & 17 & 4 & 5 & 4,0 \\
\hline 11 & 27 & 60 & 10 & 5 & 4 & 4,8 \\
\hline 12 & 25 & 120 & 8 & 2 & 6 & 6,4 \\
\hline 13 & 25 & 150 & 8 & 1 & 8 & 3,6 \\
\hline 14 & 30 & 140 & 13 & 5 & 7 & 8,5 \\
\hline 15 & 12 & 60 & 11 & 2 & 5 & 5,0 \\
\hline 16 & 28 & 70 & 7 & 1 & 7 & 9,3 \\
\hline 17 & 15 & 70 & 25 & 2 & 5 & 2,1 \\
\hline 18 & 12 & 50 & 8 & 1 & 9 & 2,9 \\
\hline 19 & 15 & 100 & 17 & 3 & 4 & 5,5 \\
\hline 20 & 30 & 90 & 8 & 2 & 9 & 2,1 \\
\hline 21 & 25 & 40 & 8 & 3 & 7 & 7,0 \\
\hline 22 & 20 & 80 & 14 & 2 & 4 & 5,1 \\
\hline 23 & 20 & 80 & 18 & 1 & 4 & 4,5 \\
\hline Mean & 22,6 & 90.1 & 14,7 & 2,2 & 6,2 & \\
\hline$S D$ & 8,6 & 30.8 & 10,00 & 1,2 & 2,0 & \\
\hline & & & & & & 7 \\
\hline
\end{tabular}

